\title{
CASE REPORT \\ A RARE CASE OF DEXTROCARDIA AND SITUS INVERSUS WITH TETRALOGY OF FALLOT IN A 23-YEAR-OLD FEMALE: A CASE REPORT
}

\author{
Zara Shirazi $^{1}$, Sohail Bangash', Saad Badar', Iqbal Pathan', Abdul Wasay ${ }^{1}$ \\ ${ }^{1}$ National Institute of Cardiovascular Diseases (NICVD), Karachi, Pakistan
}

Tetralogy of Fallot (TOF) is commonly identified cyanotic congenital cardiac anomaly; however, its affiliation with dextrocardia and situs inversus is very rare. We describe a case of dextrocardia and situs inversus associated with TOF in a 23 -year old girl who arrived with central shunt at the age of 17 years and later for total surgical correction with magnificent outcomes.

Keywords: congenital heart defects, dextrocardia, tetralogy of fallot, cardiac surgical procedure, septal defects

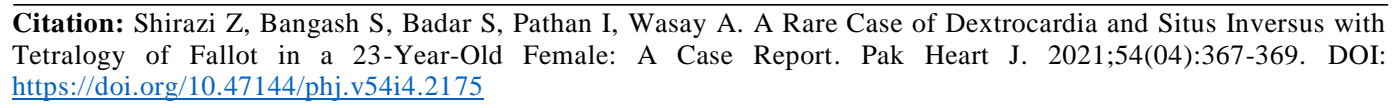

\section{INTRODUCTION}

The most usually congenital cyanotic cardiac anomaly Tetralogy of Fallot (TOF) comprises of obstruction of right ventricular outflow tract (RVOT), overriding of aorta, ventricular septum defect and hypertrophy of concentric right ventricle. ${ }^{1}$ Secundum atrial septal defect or right aortic arch are common concomitant defects that are sometimes correlated. Tetralogy of Fallot (TOF) with complete situs ambiguous or inversus is rarely described and come across with technical challenges in intracardiac repair.

Surgical correction is the treatment of choice of TOF and has enhanced the outcomes significantly.,3 However, late diagnosis and delayed surgical correction are mostly linked with substandard end results. ${ }^{4}$

The case represents the challenges associated with diagnosing and treatment of such cases. We describe a rare case of dextrocardia and situs inversus associated with TOF in a 23-year-old girl who came to our institute and done with central shunt at the age of 17 years and then after three years for total surgical correction with good end results.

\section{CASE REPORT}

A 23-year-old female had been diagnosed with dextrocardia with situs inversus associated with tetralogy of fallot at the age of 19 years. She had experienced decreased exercise tolerance, recurrent episodes of cyanosis and squatting, and marked shortness of breath upon working and exertion throughout her life. No complaints of palpitations or chest pain.
In November 2017, a central shunt was done. Polytetrafluoroethylene (PTFE) shunt was placed between ascending aorta and main pulmonary artery in end to side fashion. Central shunt was done because there were poor peripheral atborization in the lungs therefore total correction was not the option at that time. Status post shunt, she was planned for total correction. At presentation she was 23 years old. For her age her body weight was 30 kilograms, and her height was 150 centimeters. On physical exam there was cyanosis, grade 2 clubbing and scleral hyperemia. The patient's hemoglobin at presentation was 15 $\mathrm{mg} / \mathrm{dl}$. Result of pulmonary exam was normal, and no organomegaly was detected. Dextrocardia was remarkable on 15-lead electrocardiogram (Figure 1). Dextrocardia with situs inversus was also shown in chest radiographs with normal heart size limits (Figure 2A).

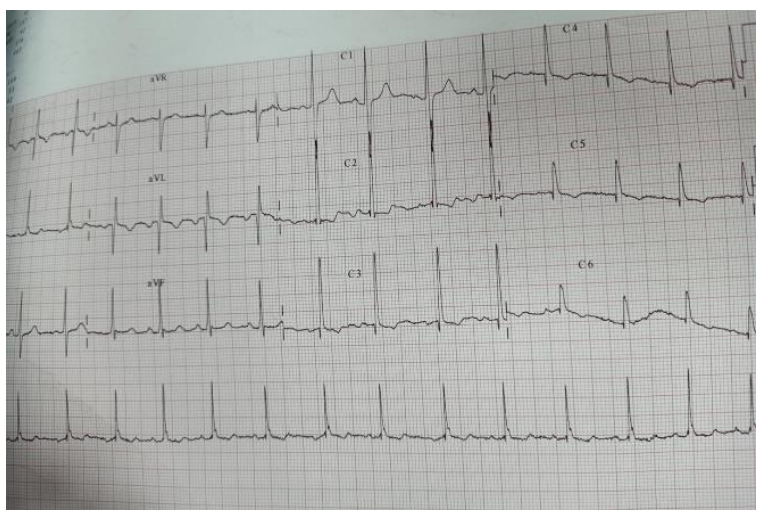

Figure 1: Surface electrocardiogram shows a pattern consistent with dextrocardia 

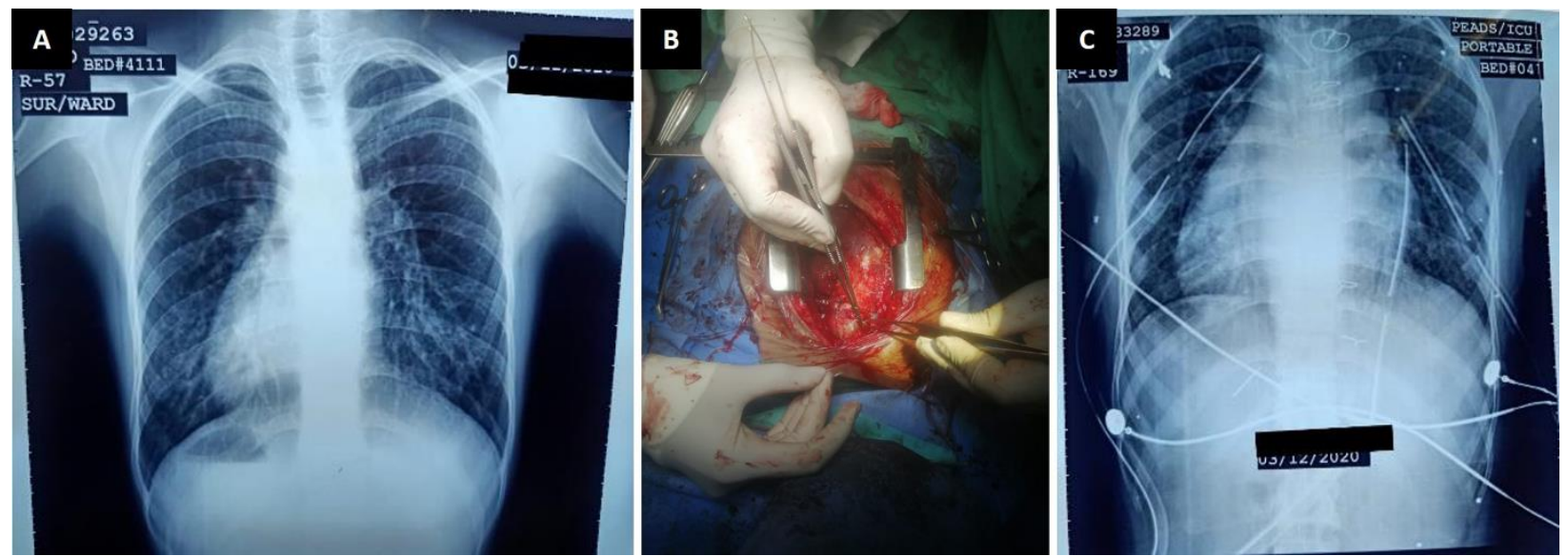

Figure 2: Chest $X$-ray shows dextrocardia (A), intraoperative picture showing surgical correction (B), and chest $X$-ray shows dextrocardia post operatively $(C)$

The anatomy of dextrocardia with situs inversus and TOF was revealed by transthoracic echocardiogram: ventricular septal defect $20 \mathrm{~mm}$ with obstruction of right ventricular outflow tract and an overriding aorta. $12 \mathrm{~mm}$ was the diameter of pulmonary valvular annulus and the sizes of left and right pulmonary arteries were 9.5 and 10 respectively with no peripheral pulmonary artery stenosis. RV Systolic and dystolic mild dysfunction was present. Atrial septal defect (ASD) II present. Z scoring for main pulmonary artery, right pulmonary artery and left pulmonary artery were $-4.53,-1.38$ and -0.85 respectively. The heart rate of patient preoperatively was 95 beats per minute; her blood pressure was measured 109/72 $\mathrm{mmHg}$. Transcutaneous oxygen saturation on room air was $89 \%$.

Our patient underwent surgical correction. According to the technique with aortic and venous dual drainage of right atrium, cardiopulmonary bypass (CPB) was established (Figure B). There were some practical difficulties during CPB and correction but approach through right atrium (RA) easily get done with the ventricular septal defect patch repair. For such kind of complex congenital diseases, approach through RA seems much beneficial than right ventricle (RV).

After pericardiotomy, an approach that is combined including both a trans atrial/transpulmonary to reconstruct the RVOT musculature that were causing outflow hindrance. There is always a possibility of damaging the conduction system or the tricuspid valvular apparatus which should be kept down.

A bovine pericardial patch was used to close the ventricular septal defect (VSD) as well as a transannular patch for relieving RVOT obstruction. 109 minutes was the cardiopulmonary bypass time while that of 84 minutes was the aortic cross-clamp time.
After uneventful weaning from cardiopulmonary bypass, pulmonary pressure gradient was measured that was $17 \mathrm{~mm} \mathrm{Hg}$.

There were no events in the postoperative period and the patient discharged on $6^{\text {th }}$ operative day. Before discharge echocardiography was done which showed the complete closure of VSD with no remnant shunting, situs inversus, dextrocardia, no RVOT obstruction and mild RV dysfunction (Figure 2C). There was no regurgitation and the pulmonary valve was functioning normally.

\section{DISCUSSION}

TOF with concomitant dextrocardia and situs inversus is very much infrequent. In 1952 Scragg and Denny first described this combination and there are only few reports on this defect. ${ }^{5}$ In one study of 147 TOF patients who were adult, there were $2(1.4 \%)$ patients with dextrocardia. ${ }^{6}$ There was another study in which 63 patients were included with dextrocardia in which one was with situs inversus and TOF (1.6\% prevalence). ${ }^{7}$

There is an advancement going on in diagnostic criteria and the conditions are better understandable in today's era if we compare with the previous centuries but still there are some late presentations reported. Reasons behind TOF delayed diagnoses can be due to less acute symptoms and far approach of better health facility.

Tetralogy of Fallot consists of overriding aorta, VSD, RV hypertrophy and obstruction of RV outflow tract. ${ }^{8}$ If we see towards the last decades, surgical rectifying is mostly being done and it was done in a fashion of 2stages: first shunt that is palliative arterial systemic-topulmonary, and then followed with repair (age range for a patient was 3-5 years) closure of VSD and 
reconstruction of the RV outflow passage. ${ }^{8,9}$ Nowadays, if symptoms appear a baby of around 3 to 6 months old, or even earlier, single-staged complete repair is proposed as well. ${ }^{8,9}$ An approach through trans atrial-transpulmonary with reconstruction of the musculature through the right atrium and pulmonary artery has replaced the classical trans-ventricular approach. This substitute procedure decreases the ventricular incision length and reduce the occurrence of ventricular arrhythmias, ventricular dysfunction with aneurysm formation. ${ }^{8}$

The challenges face by the surgeon is mostly due to altered anatomical location and orientation of the heart as well as visualization and closure of the VSD and relief of RVOT obstruction. This atypical arrangement may make it more difficult to repair the defects (VSD closure, RVOT, and pulmonary artery extension). Therefore, the risk of residual VSD as well as RVOT obstruction and damage to the conduction system may also be higher. ${ }^{10}$

Surgeon's technique of choice depends on the size of pulmonary valvular annulus and the location of the anomalous coronary artery. Transatrial/transpulmonary approach is usually preferred technique that is used whenever possible. ${ }^{11}$ Echocardiogram, computed tomography (CT) scan of the chest and magnetic resonance imaging (MRI) of the chest usually helps in evaluation for dextrocardia and associated anomalies. ${ }^{12}$

The end result of managing congenital cardiac diseases in dextrocardia patients depend on the difficulty and complex cardiac anomalies. As the dextrocardia is diagnosed initially, patients can be treated by physicians similarly as with TOF and dextrocardia.

In conclusion, our patient had a combination of TOF, dextrocardia with situs inversus. Complete surgical correction was carried out using combination of approaches. Challenges came for the surgeon but with

\section{Address for Correspondence:}

Dr. Zara Shirazai, Resident at Department of Cardiac Surgery, National Institute of Cardiovascular Diseases, Karachi, Pakistan.

Email: zaramirza_fd@hotmail.com proper techniques VSD margins were closed and total correction was carried out. Avoiding damage to the atrioventricular node and penetrating bundle was the main concern which was successfully tackled with such anatomy.

\section{AUTHORS' CONTRIBUTION}

ZS: Concept and design, data acquisition, interpretation, drafting, final approval, and agree to be accountable for all aspects of the work. SoB, SaB, IP, AW: Data acquisition, interpretation, drafting, final approval and agree to be accountable for all aspects of the work.

Conflict of interest: Authors declared no conflict of interest.

\section{REFERENCES}

1. Muacevic A, Adler J, Alkashkari W, Al-Husayni F, Almaqati A, AlRahimi J, et al. An Adult Patient with a Tetralogy of Fallot Case. Cureus. 2020;12(11):e11658.

2. Gatzoulis MA, Balaji S, Webber SA, Siu SC, Hokanson JS, Poile $\mathrm{C}$, et al. Risk factors for arrhythmia and sudden cardiac death late after repair of Tof: a multicenter study. Lancet. 2000;356:975-81.

3. Geva T. Indications and timing of pulmonary valve replacement after Tof repair. Semin Thorac Cardiovasc Surg Pediatr Card Surg Annu. 2006;9:11-22.

4. Bernier PL, Stefanescu A, Samoukovic G, Tchervenkov CI. The challenge of congenital heart disease worldwide: epidemiologic Card Surg Annu. 2010;13:26-34.

5. Scragg JN, Denny M. Dextrocardia, tetralogy of Fallot, and situs inversus; report of a case. S Afr Med J. 1952;26:1025-8.

6. Abraham KA, Cherian G, Rao VD, Sukumar IP, Krishnaswami S, John S. Tetralogy of Fallot in adults. A report on 147 patients. Am J Med. 1979;66:811-6.

7. Evans WN, Acherman RJ, Collazos JC, Castillo WJ, Rollins RC, Kip KT, et al. Dextrocardia: practical clinical points and comments on terminology. Pediatr Cardiol. 2010;31:1-6.

8. Apitz C, Webb GD, Redington AN. Tetralogy of Fallot. Lancet. 2009;374:1462-71.

9. Starr JP. Tetralogy of Fallot: yesterday and today. World J Surg. 2010;34:658-68

10. Dilorenzo M, Weinstein, S, Shenoy R. Tetralogy of fallot with dextrocardia and situs inversus in a 7-year-old boy. Tex Heart Inst J. 2013;40(4):481-3

11. Giordano, R . Surgical strategy for tetralogy of Fallot with abnormal coronary arteries. J Thorac Dis. 2017;9(10):3447-9

12. Esmaeil H, Al-Fadhli J, Dashti A, Al-Sarraf N. Ischemic mitral regurgitation in a patient with dextrocardia and situs inversus totalis. J Surg Case Rep. 2019;2019(11):rjz329. and demographic facts. Semin Thorac Cardiovasc Surg Pediatr 